Research Article

\title{
Electromagnetic Scattering by Multiple Columns Partially Buried in a Ground Plane
}

\author{
Xin-Cheng Ren, Ye Zhao, Peng-Ju Yang, and Xiao-Min Zhu \\ School of Physics and Electronic Information, Yanan University, Yanian 716000, China \\ Correspondence should be addressed to Peng-Ju Yang; pjyang@yeah.net
}

Received 22 May 2017; Revised 1 October 2017; Accepted 8 October 2017; Published 1 November 2017

Academic Editor: Atsushi Mase

Copyright (c) 2017 Xin-Cheng Ren et al. This is an open access article distributed under the Creative Commons Attribution License, which permits unrestricted use, distribution, and reproduction in any medium, provided the original work is properly cited.

\begin{abstract}
Wideband electromagnetic scattering from multiple objects partially buried beneath rough earth soil surfaces is an important topic during recent years due to its extensive applications in several fields, such as ground remote sensing, ground penetrating radar applications, and target identification. Due to the advantages of finite-difference time-domain (FDTD) method in calculating the wideband electromagnetic scattering from rough surface in the presence of multiple objects, the FDTD method under ultrawideband (UWB) Gaussian pulse wave incidence is utilized in the present study for analyzing the frequency response of rough soil surfaces with periodically distributed multiple rectangular cross-section columns buried partially. In this paper, the dielectric property of the actual land surface is expressed using the four-component model, and the actual rough land surface is simulated utilizing Monte Carlo method with exponential correlation function. The emphasis of the present study is on analyzing the wideband response signatures of composite backscattering coefficient varying with frequency on the basis of extensive numerical simulations, in particular for calculating and discussing in detail the influence of the root-mean-square height and the correlation length of rough soil surface, soil moisture, the length and the width of the rectangular cross-section column, separation distance, burial depth, and tilt angle on the composite backscattering coefficient.
\end{abstract}

\section{Introduction}

During recent years, more and more attentions are paid to the electromagnetic (EM) wave scattering from rough surface and composite scattering from rough surface with target because of their spread application [1-5] in environmental remote sensing, target tracking and recognition, radar detection, wireless communication, and microwave probing of composite materials and their surface roughness. In particular, the short pulses and ultrawideband radar are widely used in target identification and remote sensing [6]. Ultrawideband radar needs the wideband RCS of target to achieve long-range detection and tracking stability [7]. The methods of solving scattering problems are plenty, such as the Kirchhoff approximation (KA) [8], small perturbation method (SPM) [9], small slope approximation (SSA) [10], the Method of Moment (MoM) [11], the Finite-Difference TimeDomain (FDTD) [12], and finite element method (FEM) [13]. In previous studies, the composite scattering from the rough sea surface and single target under harmonic wave irradiation have been reported and various methods are used in these studies [14-20]. However, the scattering from rough land surface such as soil surface, sand surface, and periodic distributed target under pulse wave irradiation was considered rarely [21, 22].

As a numerical method, FDTD can calculate the electromagnetic scattering problems under pulse wave and has superiority of the time-domain numerical algorithm [23-25]. In this paper, we consider the characteristics of composite wideband electromagnetic scattering from rough soil surface and the partially buried periodic distributed rectangular cross-section columns under Gaussian pulse wave irradiation using FDTD, and the frequency response curves of back composite scattering coefficient are obtained. The results of numerical calculation have a very great importance for achieving the remote sensing information of the earth under the complex electromagnetic environment. 


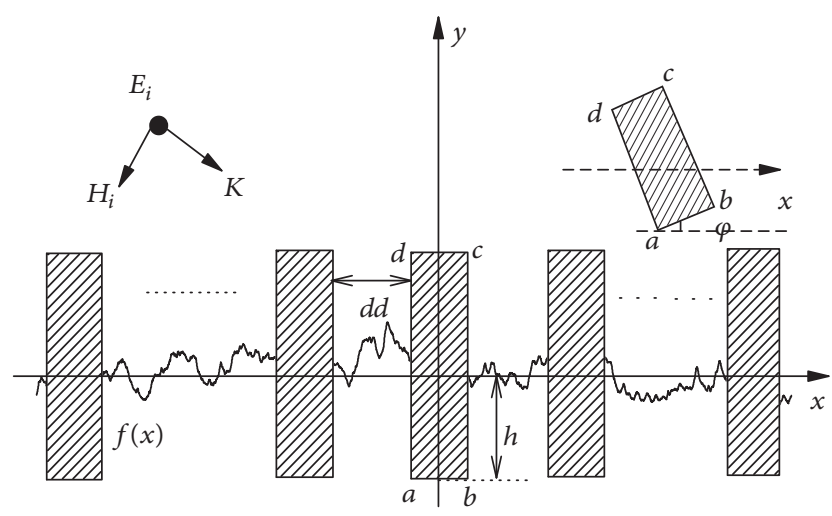

Figure 1: Geometry of composite scattering from the rough soil surface and partially buried periodic distributed rectangular crosssection columns.

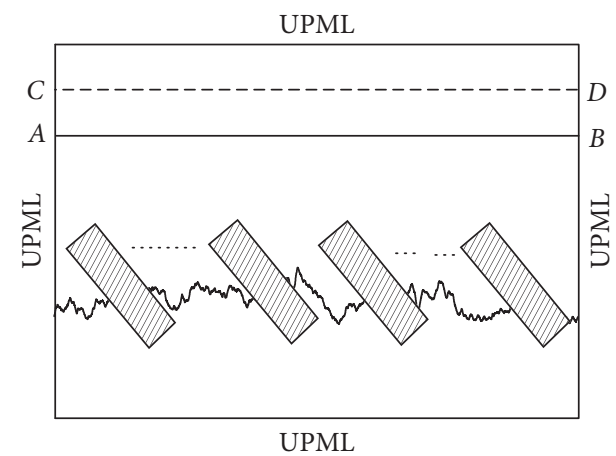

FIGURE 2: FDTD computation in the composite scattering model from the rough surface and partially buried periodic distributed rectangular cross-section columns.

\section{Composite Scattering Model and FDTD Method}

In the present study, the actual rough soil surface $f(x)$ is simulated utilizing Monte Carlo method [26] with 1D exponential. The scattering geometry in this paper is illustrated in Figure 1.

Suppose that the upper of the coordinate axis $x$ is homogeneous free space and that the lower is isotropic homogeneous soil medium. Periodic distributed rectangular cross-section columns are labeled as $a b c d$, which are partially buried in soil. The separation distance of every two adjacent columns is expressed as $d d . h$ is the burial depth, and $\varphi$ represents tilt angle, which is the intersection angle of the side length $a b$ of column and the forward of coordinate axis $x$.

As shown in Figure 2, the calculated area of composite scattering model is built by FDTD. In the area, it is divided into scattering field region and total region by setting up adjacent boundary $A B$, at the same time, setting up extrapolated boundary $C D$ in the scattering field region. These two boundaries are planes and extended to absorption boundary. In order to absorb incident wave, we set up absorption boundary conditions which are made of anisotropic medium perfectly matched layer (PML) of thickness of ten grids outside the area, then selecting Gaussian pulse electromagnetic wave as incident wave, and total region from adjacent boundary $A B$ is introduced.

The actual rough land surface is modeled as realizations of a Gaussian random process with exponential correlation function by using Monte Carlo method [26] and the dielectric constant of soil using the four-component model established by Wang and Schmugge [27] in this work.

The power spectral density of exponential correlation rough surface is expressed as [28]

$$
S(k)=\frac{\delta^{2} l}{\pi\left(1+k^{2} l^{2}\right)},
$$

where $\delta$ and $l$ are the root-mean-square and correlation length of rough soil surface, respectively. $k$ is space wave number.

The rough soil surface is simulated by Monte Carlo method, and 1D rough surface realization with length $L$ can be expressed as

$$
f\left(x_{n}\right)=\frac{1}{L} \sum_{j=0}^{j=N-1} F\left(k_{j}\right) \cdot \exp \left(i k_{j} x_{n}\right),
$$

where $x_{n}=n \Delta x(n=1, \ldots, N)$, and it represents the $n$th discrete points of rough surface. $k_{j}=2 \pi j / L$ is space wave number. $F\left(k_{j}\right)$ and $f\left(x_{n}\right)$ are Fourier's transformation pairs. $F\left(k_{j}\right)$ is defined as

$$
\begin{aligned}
F\left(k_{j}\right)= & \frac{2 \pi}{\sqrt{2 \Delta K}} \sqrt{S\left(k_{j}\right)} \\
& \cdot \begin{cases}{[N(0,1)+i N(0,1)]} & j=1, \ldots, \frac{N}{2}-1 \\
N(0,1) & j=0, \frac{N}{2},\end{cases}
\end{aligned}
$$

where $\Delta K$ is space wave number difference of wave spectrum sample adjacent spectral domain, and $N(0,1)$ represents a Gaussian random variable that the mean is 0 and the variance is 1 . When $j>N / 2$, then $F\left(k_{j}\right)=F\left(k_{N-j}\right)^{*}$, and this ensures that the rough surface profile obtained by inverse Fourier transform is a real number.

The dielectric constant of the soil is mainly affected by the incident frequency $f$, soil moisture $m_{V}$, soil surface temperature $T$, soil type, and other factors. A four-component model is established by Wang and Schmugge, assuming the sand content in soil is $S$, clay content in soil is $C$, and then the humidity compression point of soil $W_{P}$ is

$$
W_{P}=0.06774-0.00064 S+0.00478 C \text {. }
$$

The critical body humidity of soil is

$$
m_{t}=0.49 W_{p}+0.165
$$

Assuming that the rock density of soil is $\rho_{r}$ and dry soil density is $\rho_{b}$, then the plot porosity of soil $p$ is

$$
p=1-\frac{\rho_{b}}{\rho_{r}} .
$$


Under normal circumstances, the rock density of soil is $\rho_{r}=$ $2.6 \mathrm{~g} / \mathrm{cm}^{3}$, and $\rho_{b}$ is determined by the following formula:

$$
\begin{aligned}
\rho_{b} & =\frac{3.455}{R^{0.3018}} \\
R & =25.1-0.21 C+0.22 C
\end{aligned}
$$

When $m_{V} \leq m_{t}$, the effective dielectric constant of the soil is

$$
\varepsilon=m_{V} \varepsilon_{x}+\left(p-m_{V}\right) \varepsilon_{a}+(1-p) \varepsilon_{r}
$$

where

$$
\begin{gathered}
\varepsilon_{x}=\varepsilon_{i}+\left(\varepsilon_{W}-\varepsilon_{i}\right) \beta \frac{m_{V}}{m_{t}}, \\
\beta=-0.57 W_{P}+0.481,
\end{gathered}
$$

where $\varepsilon_{a}=1.0$ is dielectric constant of air and $\varepsilon_{i}=3.15-$ $i 0.025$ is dielectric constant of ice. $\varepsilon_{W}$ is dielectric constant of pure water and can be calculated by the Debye equation; namely,

$$
\begin{aligned}
\varepsilon_{W}= & 4.9+\frac{\varepsilon_{W 0}-4.9}{1+i 2 \pi f \tau_{w}} \\
\varepsilon_{W 0}= & 88.045-0.4147 T+6.295 \times 10^{-4} T^{2}+1.705 \\
& \times 10^{-5} T^{3} \\
2 \pi \tau_{w}= & 1.1109 \times 10^{-10}-3.824 \times 10^{-12} T+6.938 \\
& \times 10^{-14} T^{2}-5.096 \times 10^{-16} T^{3}
\end{aligned}
$$

In (11)-(12), $T$ denotes temperature. When $m_{V}>m_{t}$, the effective dielectric constant of the soil is

$$
\begin{aligned}
\varepsilon= & m_{t} \varepsilon_{x}+\left(m_{V}-m_{t}\right) \varepsilon_{W}+\left(p-m_{V}\right) \varepsilon_{a} \\
& +(1-p) \varepsilon_{r}, \\
\varepsilon_{x}= & \varepsilon_{i}+\left(\varepsilon_{W}-\varepsilon_{i}\right) \beta .
\end{aligned}
$$

Maxwell's equations are a set of basic equations that govern the macroscopic electromagnetic phenomena. For two-dimensional electromagnetic scattering problems, rectangular components of the electromagnetic field may be classified as independent groups, namely, TM wave and TE wave. In TM wave, as an example, FDTD difference equation has the following form [29]:

$$
\begin{aligned}
& H_{x}^{n+1 / 2}\left(p, q+\frac{1}{2}\right)=C P(m) H_{x}^{n-1 / 2}\left(p, q+\frac{1}{2}\right) \\
& -C Q(m) \frac{E_{z}^{n}(p, q+1)-E_{z}^{n}(p, q)}{\Delta y}, \\
& H_{y}^{n+1 / 2}\left(p+\frac{1}{2}, q\right)=C P(m) H_{y}^{n-1 / 2}\left(p+\frac{1}{2}, q\right) \\
& +C Q(m) \frac{E_{z}^{n}(p+1, q)-E_{z}^{n}(p, q)}{\Delta x},
\end{aligned}
$$

$$
\begin{aligned}
& E_{z}^{n+1}(p, q)=C A(m) E_{z}^{n}(p, q)+C B(m) \\
& \cdot\left[\frac{H_{y}^{n+1 / 2}(p+1 / 2, q)-H_{y}^{n+1 / 2}(p-1 / 2, q)}{\Delta x}\right.
\end{aligned}
$$

$$
\left.-\frac{H_{x}^{n+1 / 2}(p, q+1 / 2)-H_{x}^{n+1 / 2}(p, q-1 / 2)}{\Delta y}\right] \text {, }
$$

where $\varphi=40^{\circ}, \sigma$ are discrete grid width along the $\delta=$ $1 \mathrm{~cm}, D=1.6$ is direction of FDTD computational domain, respectively, and the coefficients $C A, C B, C P$, and $C Q$ are, respectively,

$$
\begin{aligned}
& C A(m)=\frac{\varepsilon(m) / \Delta t-\sigma(m) / 2}{\varepsilon(m) / \Delta t+\sigma(m) / 2}, \\
& C B(m)=\frac{1}{\varepsilon(m) / \Delta t+\sigma(m) / 2}, \\
& C P(m)=\frac{\mu(m) / \Delta t-\sigma_{m}(m) / 2}{\mu(m) / \Delta t+\sigma_{m}(m) / 2}, \\
& C Q(m)=\frac{1}{\mu(m) / \Delta t+\sigma_{m}(m) / 2},
\end{aligned}
$$

where the value of grade $m$ and the left field component node labels have the same position. In (15), $\sigma(m)$ denotes the electric conductivity. $\varepsilon(m)$ represents electrical permittivity. $\mu(m)$ stands for the magnetic permeability. $\Delta t$ is the time increment

FDTD calculation is only in a limited area. Thus, it is necessary to set absorbing boundary in truncated boundary for open domain electromagnetic scattering problem. Anisotropic perfectly matched layer (UPML) absorbing boundary is selected in this work. FDTD iteration order of UPML layers is $H_{x}, H_{y} \rightarrow P_{z}^{\prime} \rightarrow P_{z} \rightarrow E_{z}$ and $E_{z} \rightarrow$ $B_{x}, B_{y} \rightarrow H_{x}, H_{y}$, and the iterative formula of electronic field $E_{z}$ of UPML layer of truncated conductive medium is below:

$$
\begin{array}{r}
P_{z}^{\prime n+1}(p, q)=C A(m) P_{z}^{\prime n}(p, q)+C B(m) \\
\cdot\left[\frac{H_{y}^{n+1 / 2}(p+1 / 2, q)-H_{y}^{n+1 / 2}(p-1 / 2, q)}{\Delta x}\right.
\end{array}
$$




$$
\begin{aligned}
& \left.-\frac{H_{x}^{n+1 / 2}(p, q+1 / 2)-H_{x}^{n+1 / 2}(p, q-1 / 2)}{\Delta y}\right], \\
& P_{z}^{n+1}(p, q)=C 1(m) P_{z}^{n}(p, q)+C 2(m) \\
& \quad \cdot\left[P_{z}^{\prime n+1}(p, q)-P_{z}^{\prime n}(p, q)\right], \\
& E_{z}^{n+1}(p, q)=C 3(m) E_{z}^{n}(p, q)+C 4(m) \cdot\left[P_{z}^{n+1}(p, q)\right. \\
& \left.\quad-P_{z}^{n}(p, q)\right],
\end{aligned}
$$

where the coefficients $C A(m)$ and $C B(m)$ are the same with $(8)$ and the coefficients $C 1(m), C 2(m), C 3(m)$, and $C 4(m)$ are

$$
\begin{aligned}
& C 1(m)=\frac{\kappa_{x}(m) / \Delta t-\sigma_{x}(m) / 2 \varepsilon_{0}}{\kappa_{x}(m) / \Delta t+\sigma_{x}(m) / 2 \varepsilon_{0}}, \\
& C 2(m)=\frac{1 / \Delta t}{\kappa_{x}(m) / \Delta t+\sigma_{x}(m) / 2 \varepsilon_{0}}, \\
& C 3(m)=\frac{\kappa_{y}(m) / \Delta t-\sigma_{y}(m) / 2 \varepsilon_{0}}{\kappa_{y}(m) / \Delta t+\sigma_{y}(m) / 2 \varepsilon_{0}}, \\
& C 4(m)=\frac{1 / \Delta t}{\kappa_{y}(m) / \Delta t+\sigma_{y}(m) / 2 \varepsilon_{0}},
\end{aligned}
$$

where $\varepsilon_{0}$ is dielectric constant in vacuum and the value of $\sigma_{w}$ and $\kappa_{w}(w=x, y)$ is

$$
\begin{aligned}
& \sigma_{w}(w)=\sigma_{w, \max } \frac{\left|w-w_{0}\right|^{m}}{d^{m}}, \\
& \kappa_{w}(w)=1+\left(\kappa_{w, \max }-1\right) \frac{\left|w-w_{0}\right|^{m}}{d^{m}},
\end{aligned}
$$

where $d$ represents the thickness of the UPML layer and $w_{0}$ denotes the interface location that UPML layer is near FDTD area. When $m=4, \sigma_{\max }=(m+1) / \sqrt{\varepsilon_{r}} 150 \pi \delta, \kappa_{\max }=5 \sim 11$, and the absorption effect is optimal [30].

Near-field scattering data is obtained by the FDTD calculation. Each time field value on the boundary is recorded, and the far-zone scattered field time response is obtained using extrapolation methods of transient pulse source excitation. Then, the frequency response of scattering coefficient is obtained through substituting the scattering coefficient formula by the Fourier transform.

$$
\sigma=10 \lg (\mathrm{NRCS}) d B .
$$

Here, NRCS represents the Normalization Radar Cross Section. The NRCS is expressed as [24]

$$
\mathrm{NRCS}=\lim _{r \rightarrow \infty} \frac{2 \pi r}{L} \frac{\left|E_{s}\right|^{2}}{\left|E_{i}\right|^{2}} .
$$

Here, $r$ is distance from observation point to origin, $E_{s}$ is electric field of scattered wave, and $E_{i}$ is electric field of incident wave.
In this work, the Gaussian pulse electromagnetic wave is given by [24]

$$
E_{i}(t)=\exp \left[-\frac{4 \pi\left(t-t_{0}\right)^{2}}{\tau^{2}}\right] .
$$

Here, $\tau$ is constant and decides the pulse width, and it is set by $\tau=2 / f$. Generally, $t_{0}=0.8 \tau$.

\section{Numerical Results}

In the following calculations, the emphasis is put on the back composite scattering coefficient which varies with frequency. It should be noted that the incident wave is Gaussian pulse electromagnetic wave of TM polarization, the width of grid is $d x=0.5 \mathrm{~cm}$, incident angle is $\theta_{i}=30^{\circ}$, and sampling length of rough land surface is $L=1600 \mathrm{~cm}$. The final example is an ensemble average over 40 realizations of rough surfaces. The dielectric parameter of soil is calculated using models found by Wang and Schmugge [27]. In this part, we consider that type of soil is silty loam. Without special explanation, in numerical simulations, parameters of rectangular crosssection column are given with $a b=20 \mathrm{~cm}, b c=70 \mathrm{~cm}$, $\varphi=0^{\circ}, h=5 \mathrm{~cm}$, and $d d=50 \mathrm{~cm}$. The root-mean-square of rough surface fluctuation $\delta$ is $1 \mathrm{~cm}$, the correlation length of rough surface fluctuation $l$ is $4 \mathrm{~cm}$, and soil moisture $m_{v}$ is 0.2 .

In order to obtain the characteristics of composite wideband electromagnetic scattering from the rough soil surface with partially buried periodic distributed rectangular crosssection columns, the curves of back composite scattering coefficient which varies with frequency are studied by numerical calculation under different parameters of rough surface and column. In the following, the numerical simulations are performed on the computer with two $2.60 \mathrm{GHz}$ processors (Intel Xeon E5-2650), 16 GB Memory, Microsoft Windows 7 operation system, and the Fortran PowerStation 4.0 compiler. Under the configuration, the computational time and memory size for one realization are approximately $33 \mathrm{~min}$ and $47 \mathrm{MB}$, respectively.

Figure 3 depicts the distribution of $\sigma$ with the incident wave frequency $f$ for $\delta=1 \mathrm{~cm}, 3 \mathrm{~cm}$, and $5 \mathrm{~cm}$, respectively. It is found that the influence of $\delta$ on $\sigma$ is obvious. With the increase of $\delta$, the scattering coefficient $\sigma$ increases, except for some high frequency domain $(2.215 \mathrm{GHz}<f<3 \mathrm{GHz})$. In addition, the position and value for the peaks of $\sigma$ are nearly unchanged with $\delta$. On the other hand, with the increasing incident wave frequency $f$, the oscillation for the amplitude of curve becomes small.

To examine the scattering coefficient difference between single column case and multiple columns case, a comparison of single column case and multiple columns case is made in Figure 4. In Figure 4, the root-mean-square of rough surface is $\delta$ is $1 \mathrm{~cm}$. It can be readily observed that for multiple columns case obvious peaks exist, which can be attributed to the grating effect due to the scattering from periodically distributed rectangular columns.

In Figure 5, the dependency of $\sigma$ on $l$ is plotted. From Figure 5 , it is found that, with the increase of $l$, the scattering 


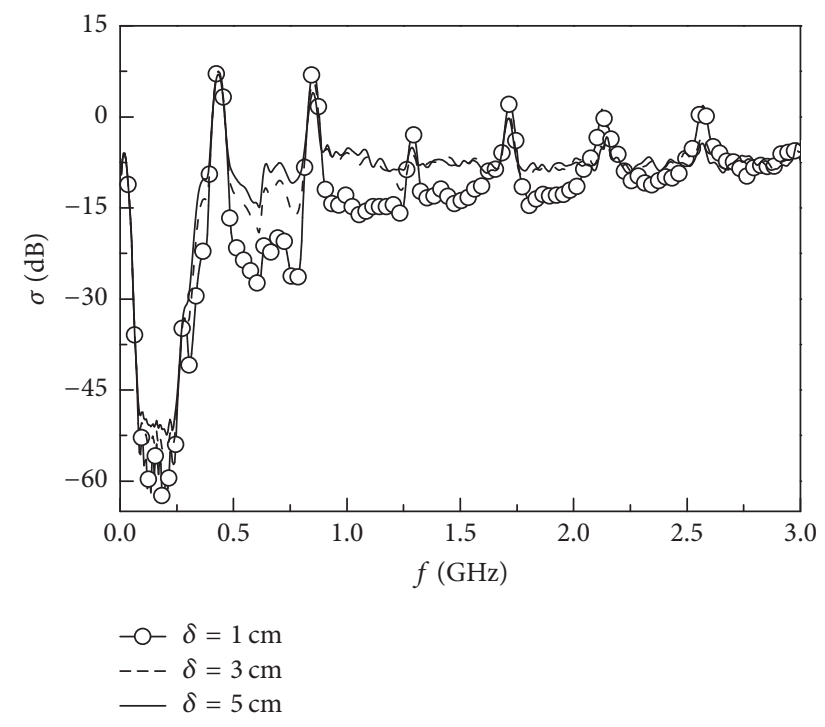

FIgURE 3: The influence of $\delta$ on $\sigma$.

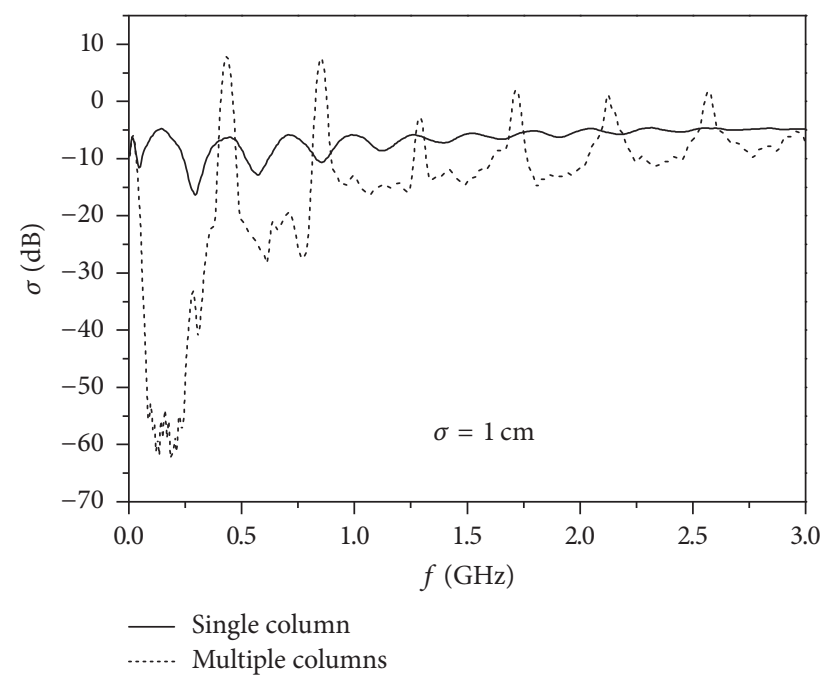

Figure 4: A comparison of single column case and multiple columns case.

coefficient $\sigma$ increases, and the position and value for the peaks of $\sigma$ are nearly unchanged with $l$.

The distribution of $\sigma$ with $f$ for different soil moisture $m_{v}$ with $\varepsilon_{r 1}=4.9301-i 0.4510\left(m_{v 1}=0.1 \mathrm{~g} / \mathrm{cm}^{3}\right), \varepsilon_{r 2}=$ $10.2802-i 1.6464\left(m_{v 2}=0.2 \mathrm{~g} / \mathrm{cm}^{3}\right)$, and $\varepsilon_{r 3}=26.0279-$ $i 5.2063\left(m_{v 3}=0.4 \mathrm{~g} / \mathrm{cm}^{3}\right)$ is depicted in Figure 6. The soil moisture represents dielectric parameter of soil. It is obvious that the influence of $m_{v}$ on $\sigma$ is small and obscure. When the frequency is less than $0.9 \mathrm{GHz}(f<0.9 \mathrm{GHz})$, all the curves in Figure 6 are coincident. However, it can be found that, with $m_{v}$ increases, $\sigma$ slightly become greater when the frequency is larger than $0.9 \mathrm{GHz}(f>0.9 \mathrm{GHz})$.

Figure 7 depicts the distribution of $\sigma$ with $f$ for different breadth of column $a b(a b=20 \mathrm{~cm}, 60 \mathrm{~cm}, 100 \mathrm{~cm}$, and $160 \mathrm{~cm}$ ). It is found that the influence of $a b$ on $\sigma$ is obvious; that is, with the increase of $a b$, the number of rectangular cross-section columns decreases. And the oscillation for the

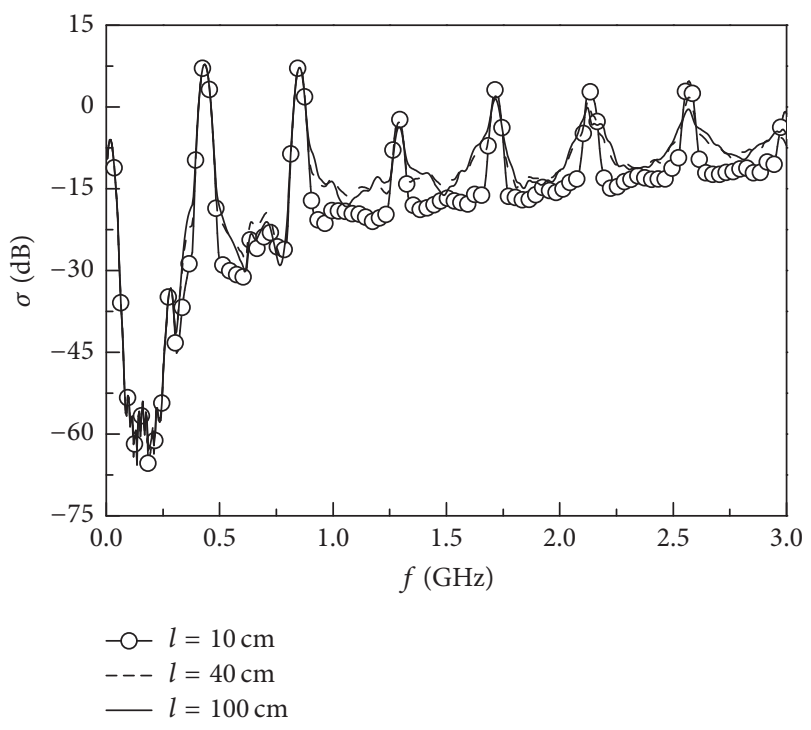

Figure 5: The influence of $l$ on $\sigma$.

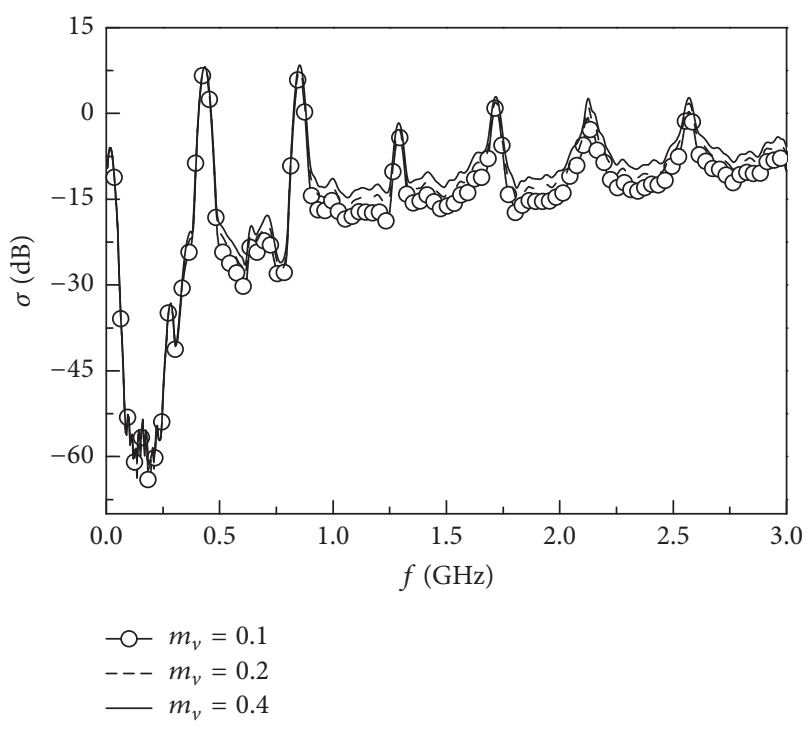

FIGURE 6: The influence of $m_{v}$ on $\sigma$.

amplitude of curve becomes smaller; the oscillation for the frequency of curve becomes greater.

Figure 8 depicts the distribution of $\sigma$ with $f$ for different length of column $b c(b c=30 \mathrm{~cm}, 70 \mathrm{~cm}$, and $100 \mathrm{~cm})$. It is found that the influence of $b c$ on $\sigma$ is complex and obvious; that is, at most of the frequency points, the larger $b c$ is, the smaller $\sigma$ is, the smaller the peak value of $\sigma$. Moreover, as $f$ increases, the oscillating amplitude of the curve is smaller.

Figure 9 depicts the distribution of $\sigma$ with $f$ for different separation distance of every two adjacent columns $d d$ ( $d d=$ $30 \mathrm{~cm}, 50 \mathrm{~cm}$, and $100 \mathrm{~cm}$ ). It is found that the influence of $d d$ on $\sigma$ is obvious. At most of the frequency points, with the increase of $d d$, the number of rectangular cross-section columns decreases in the calculated area, the oscillating frequency of curve is greater, and $\sigma$ is larger for the same frequency point. 

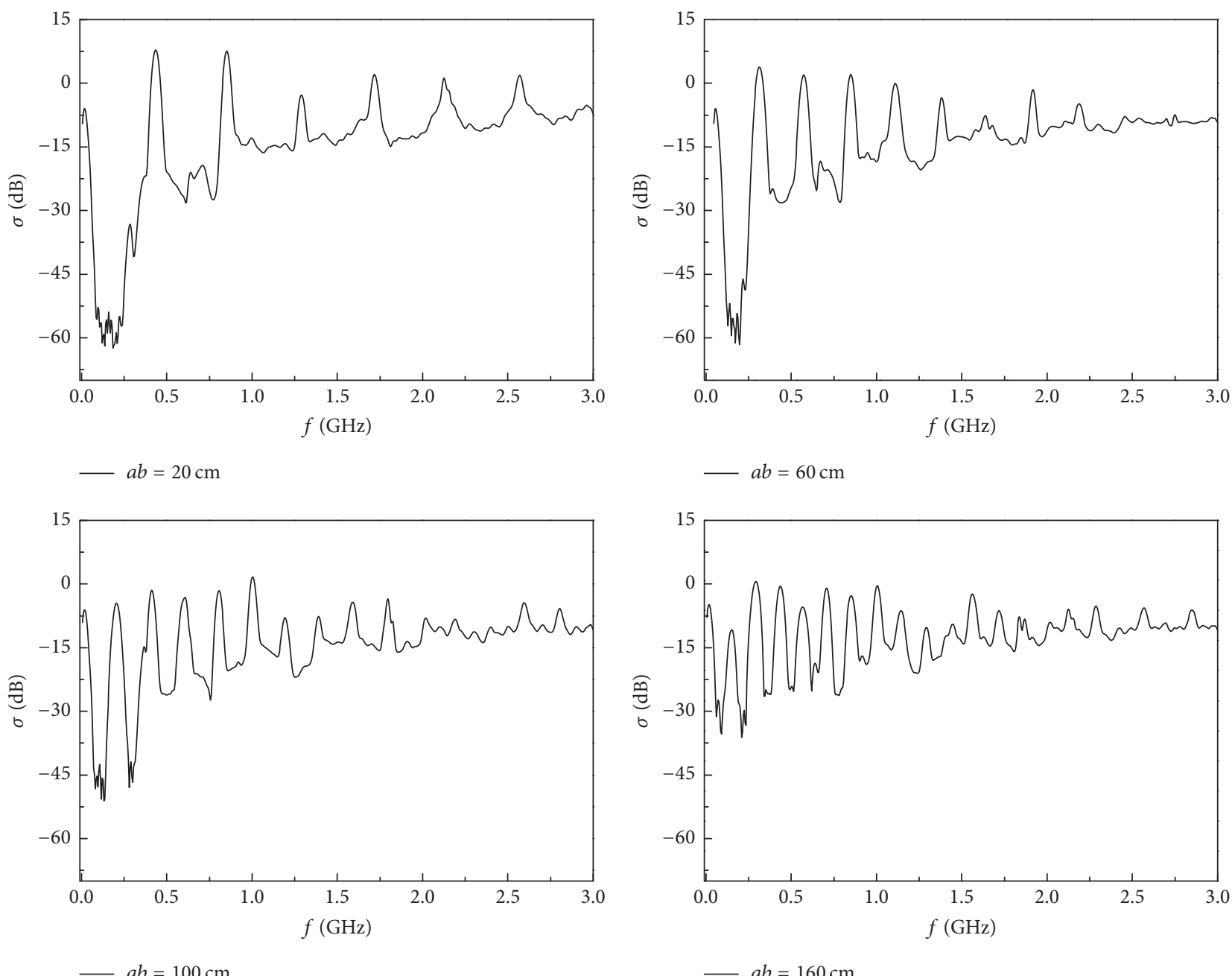

FIgURE 7: The influence of $a b$ on $\sigma$.

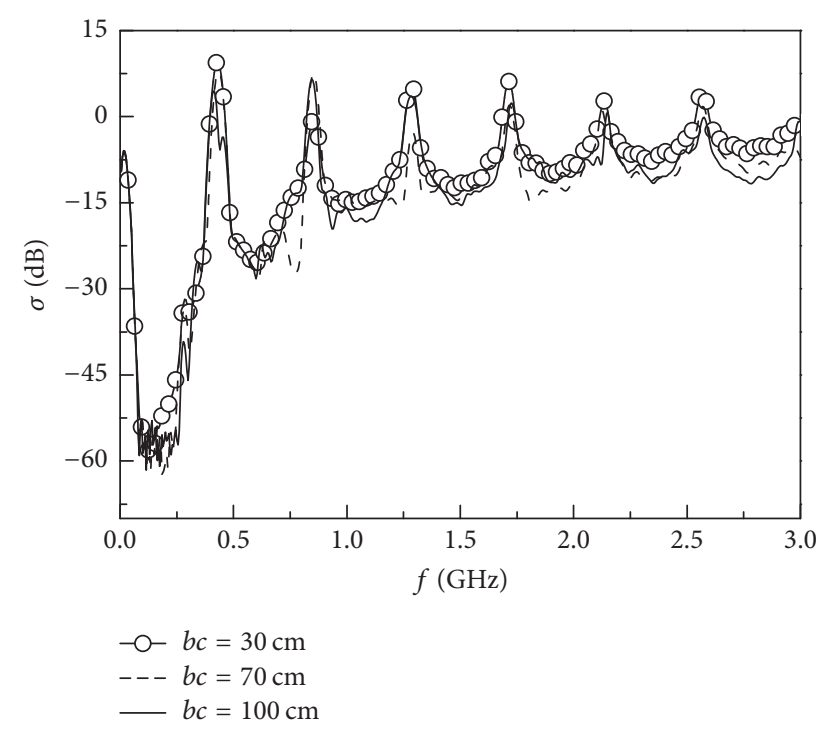

Figure 8: The influence of $b c$ on $\sigma$.

Figure 10 depicts the distribution of $\sigma$ with $f$ for different burial depth $h(h=5 \mathrm{~cm}, 15 \mathrm{~cm}$, and $25 \mathrm{~cm})$. It is obvious that the influence of $h$ on $\sigma$ is small and obscure. Part of the curves are coincident. However, it can be found that, with the increase of $h, \sigma$ becomes greater when frequency is larger than $1.73 \mathrm{GHz}(f>1.73 \mathrm{GHz})$.

Figure 11 shows the relationship of $\sigma$ with $f$ for different $\varphi\left(\varphi=10^{\circ}, 30^{\circ}\right.$, and $\left.60^{\circ}\right)$ when $h=15 \mathrm{~cm}$. Obviously, the influence of $\varphi$ on $\sigma$ is complex. When frequency is less than $1.09 \mathrm{GHz}(f<1.09 \mathrm{GHz})$, the bigger $\varphi$ is, the smaller $\sigma$ is. If frequency satisfies the inequality $(1.09 \mathrm{GHz}<f<2.5 \mathrm{GHz})$, $\sigma$ of $\varphi=30^{\circ}$ is smaller than others.

Hereinbefore, the results from Figures 3-11 in the case of TM polarization are obtained by plentiful calculation on the condition that other parameters are stationary. Only one point is worth illustrating; that is, the influence of some parameters on the back composite scattering coefficient is restricted by other parameters.

\section{Conclusion}

In this paper, the problems of composite wideband electromagnetic scattering from exponential correlation rough soil surface with partially buried periodic distributed rectangular cross-section columns under Gaussian pulse wave irradiation 


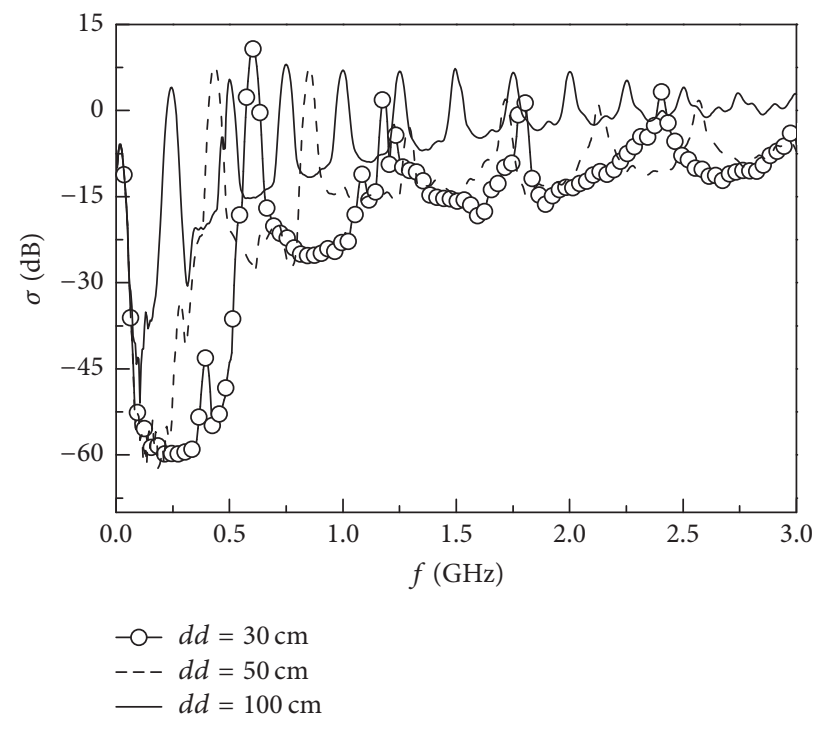

FIGURE 9: The influence of $d d$ on $\sigma$.

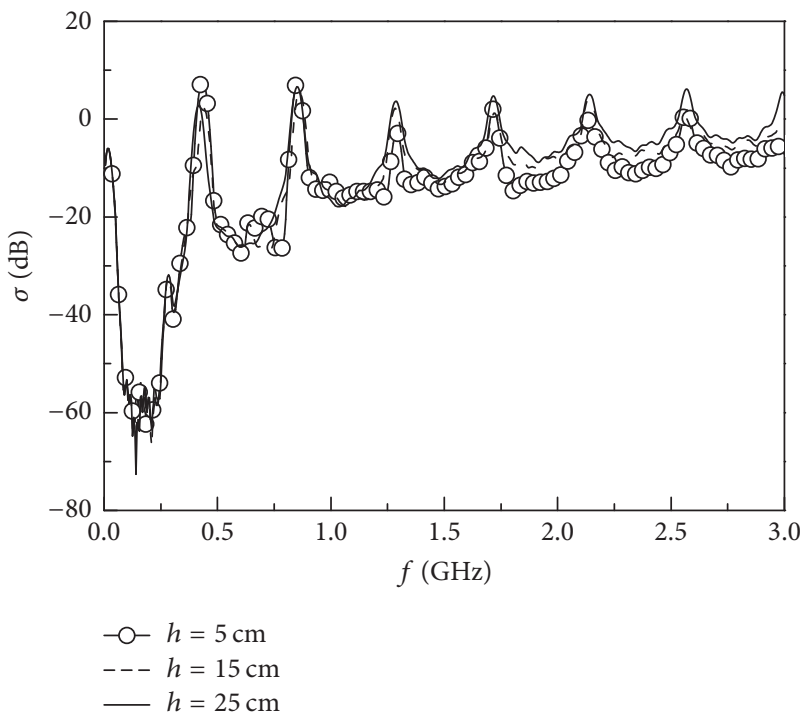

FIGURE 10: The influence of $h$ on $\sigma$.

are investigated using transient FDTD. The influence of the root-mean-square and the correlation length of rough surface fluctuation, soil moisture, the length and the width of the rectangular cross-section column, separation distance, burial depth, and tilt angle on the composite scattering signatures are discussed in detail. The numerical simulations indicate that the variation of composite scattering coefficient versus frequency is oscillatory. It is also showed that the composite scattering coefficient versus frequency increases with the increase of root-mean-square of soil surface, water ratio of soil, the target section height, and the separation distance of target. However, simulation results indicate that the composite scattering coefficient versus frequency decreases with the increase of target section width. In summary, the variation of wideband scattering coefficient is very complicated and is very sensitive to incidence angle of electromagnetic wave. However, the wideband scattering coefficient under Gaussian

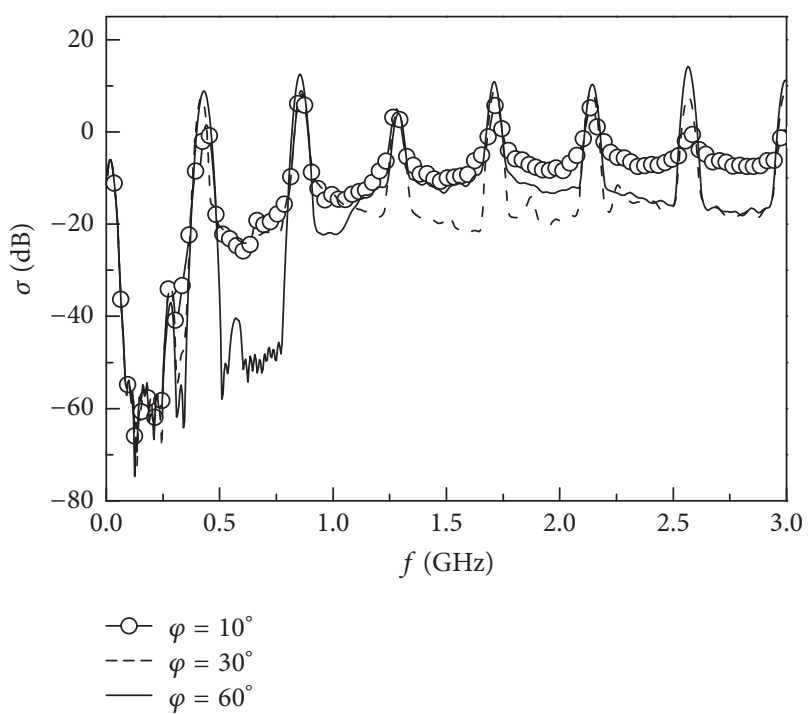

FIGURE 11: The influence of $\varphi$ on $\sigma$.

pulse wave incidence is less sensitive to the correlation length of rough soil surface, the depth of buried objects, and the dielectric constant of target. Although the multiple columns partially buried in a randomly rough surface are a rather simplified model, the present study is potentially valuable for many applications, such as ground remote sensing, ground penetrating radar applications, underground mine detection, tunnel detection, and target identification. In particular, the simplified model of periodically distributed columns represents many realistic objects, such as tree trunks, mine props, telegraph poles. The future investigation on this topic will include electromagnetic scattering from two-dimensional rough land surface with complex multiple targets.

\section{Conflicts of Interest}

The authors declare that they have no conflicts of interest.

\section{Acknowledgments}

This work was supported in part by the National Natural Science Foundation of China (Grant no. 61379026), in part by the National Science Foundation for Young Scientists of China (Grant no. 61701428), in part by the foundation of construction of high-level university project in Shaanxi Province (Grant no. 2015SXTS02), in part by the open foundation of Fudan University Key Laboratory for Information Science of Electromagnetic Waves (Grant no. EMW201502), in part by the Scientific Research Foundation of Yanan University (Grant nos. YDBK2016-17 and YDY2017-06), and in part by the Natural Science Basic Research Plan in Shaanxi Province of China (Grant no. 2014JM-6113).

\section{References}

[1] M. A. Nasr, I. A. Eshrah, and E. A. Hashish, "Electromagnetic scattering from a buried cylinder using a multiple reflection 
approach: TM case," IEEE Transactions on Antennas and Propagation, vol. 62, no. 5, pp. 2702-2707, 2014.

[2] S. Bellez, C. Bourlier, and G. Kubické, "3-D scattering from a PEC target buried beneath a dielectric rough surface: an efficient PILE-ACA algorithm for solving a hybrid KA-EFIE formulation," Institute of Electrical and Electronics Engineers. Transactions on Antennas and Propagation, vol. 63, no. 11, pp. 5003-5014, 2015.

[3] W. Yang and C. Qi, "A Bi-Iteration Model for Electromagnetic Scattering from a 3D Object above a 2D Rough Surface," Electromagnetics, vol. 35, no. 3, pp. 190-204, 2015.

[4] E. Rashidi-Ranjbar and M. Dehmollaian, "Target Above Random Rough Surface Scattering Using a Parallelized IPO Accelerated by MLFMM," IEEE Geoscience and Remote Sensing Letters, vol. 12, no. 7, pp. 1481-1485, 2015.

[5] R. W. Xu, L. X. Guo, H. J. He, and W. Liu, "A hybrid FEM/MoM technique for 3-D electromagnetic scattering from a dielectric object above a conductive rough surface," IEEE Geoscience \& Remote Sensing Letters, vol. 13, pp. 314-318, 2016.

[6] X. M. Zhu, X. C. Ren, and L. X. Guo, "Study on wide-band scattering from rectangular cross-section above rough land surface with exponential type distribution using FDTD," Acta Physica Sinica, vol. 63, 2014.

[7] A. Chehri, P. Fortier, and P. M. Tardif, "Characterization of the ultra-wideband channel in confined environments with diffracting rough surfaces," Wireless Personal Communications, vol. 62, no. 4, pp. 859-877, 2012.

[8] M. Khemiri and I. Sassi, "Regions of validity of geometric optics and Kirchhoff approximations for reflection from Gaussian random rough dielectric surfaces," Waves in random and complex media : propagation, scattering and imaging., vol. 25, no. 4, pp. 656-668, 2015.

[9] S. Afifi, R. Dusséaux, and A. Berrouk, "Electromagnetic scattering from 3D layered structures with randomly rough interfaces: Analysis with the small perturbation method and the small slope approximation," IEEE Transactions on Antennas and Propagation, vol. 62, no. 10, pp. 5200-5208, 2014.

[10] A. Berrouk, R. Dusséaux, and S. Affif, "Electromagnetic wave scattering from rough layered interfaces: Analysis with the small perturbation method and the small slope approximation," Progress in Electromagnetics Research B, no. 57, pp. 177-190, 2014.

[11] G. Apaydin, F. Hacivelioglu, L. Sevgi, and P. Y. Ufimtsev, "Wedge diffracted waves excited by a line source: Method of Moments (MoM) modeling of fringe waves," IEEE Transactions on Antennas Propagation, vol. 62, pp. 4368-4371, 2014.

[12] R. Xiong, B. Chen, J. Han, Y. Qiu, W. Yang, and Q. Ning, "Transient resistance analysis of large grounding systems using the FDTD method," Progress in Electromagnetics Research, vol. 132, pp. 159-175, 2012.

[13] O. Ozgun and M. Kuzuoglu, "Monte Carlo-based characteristic basis finite-element method (MC-CBFEM) for numerical analysis of scattering from objects on/above rough sea surfaces," IEEE Transactions on Geoscience and Remote Sensing, vol. 50, no. 3, pp. 769-783, 2012.

[14] R. Tao, Y. Li, X. Bai, and A. Waheed, "Fractional weierstrass model for rough ocean surface and analytical derivation of its scattered field in a closed form," IEEE Transactions on Geoscience and Remote Sensing, vol. 50, no. 10, pp. 3627-3639, 2012.

[15] J. Li, L.-X. Guo, S.-R. Chai, and Y.-C. Jiao, "Electromagnetic scattering from a PEC object above a dielectric rough sea surface by a hybrid PO-PO method," Waves in Random and Complex Media, vol. 25, no. 1, pp. 60-74, 2015.

[16] C. G. Jia, L. X. Guo, and P. J. Yang, "EM scattering from a target above a 1-D randomly rough sea surface using GPUbased parallel FDTD," IEEE Antennas and Wireless Propagation Letters, vol. 14, pp. 217-220, 2015.

[17] Y. Liang, L. X. Guo, Z. S. Wu, and Q. H. Liu, "A Study of Composite Electromagnetic Scattering from an Object Near a Rough Sea Surface Using an Efficient Numerical Algorithm," IEEE Antennas and Wireless Propagation Letters, vol. 15, pp. 186190, 2016.

[18] T. Wei, X. C. Ren, and L. X. Guo, "Study on composite electromagnetic scattering from the double rectangular crosssection columns above rough sea surface using hybrid method," Acta Physica Sinica, vol. 64, 2015.

[19] L.-X. Guo and R.-W. Xu, "An efficient multiregion FEM-BIM for composite scattering from an arbitrary dielectric target above dielectric rough sea surfaces," IEEE Transactions on Geoscience and Remote Sensing, vol. 53, no. 7, pp. 3885-3896, 2015.

[20] P.-J. Yang and L.-X. Guo, "Polarimetric scattering from twodimensional dielectric rough sea surface with a ship-induced Kelvin wake," International Journal of Antennas and Propagation, vol. 2016, Article ID 2474708, 2016.

[21] X. C. Ren, L. X. Guo, and Y. C. Jiao, "Investigation of electromagnetic scattering interaction between the column with rectangular cross-section and rough land surface covered with snow using finite difference time domain method," Acta Physica Sinica, vol. 61, article 144101, p. 379, 2012.

[22] R. Wang, L. X. Guo, and A. Q. Wang, "Investigation of electromagnetic scattering interaction between the buried target and the rough surface in different types of soil," Acta Phys. Sin, vol. 59, pp. 3179-3186, 2010.

[23] T. Meissner and F. J. Wentz, "The complex dielectric constant of pure and sea water from microwave satellite observations," IEEE Transactions on Geoscience and Remote Sensing, vol. 42, no. 9, pp. 1836-1849, 2004.

[24] D. Lyzenga, "Numerical simulation of synthetic aperture radar image spectra for ocean waves," IEEE Transactions on Geoscience and Remote Sensing, vol. GE-24, no. 6, pp. 863-872, 1986.

[25] R. A. Dalrymple and B. D. Rogers, "Numerical modeling of water waves with the SPH method," Coastal Engineering Journal, vol. 53, no. 2-3, pp. 141-147, 2006.

[26] C. Br Ü Ning, W. Alpers, and K. Hasselmann, "Monte-carlo simulation studies of the nonlinear imaging of a two dimensional surface wave field by a synthetic aperture radar," International Journal of Remote Sensing, vol. 11, no. 10, pp. 1695-1727, 1990.

[27] J. R. Wang and T. J. Schmugge, "An empirical model for the complex dielectric permittivity of soils as a function of water content," IEEE Transactions on Geoscience and Remote Sensing, vol. 18, no. 4, pp. 288-295, 1980.

[28] L. Tsang, J. A. Kong, and K. H. Ding, Scattering of Electromagnetic Waves: Theories and Applications, vol. 1, WileyInterscience, New York, NY, USA, 1 edition, 2000.

[29] L. Sevgi, Electromagnetic Modeling and Simulation, John Wiley \& Sons, Inc., Hoboken, NJ, USA, 2014.

[30] A. Taflove and S. C. Hagness, Computational Electrodynamics: The Finite-Difference Time-Domian Method, Artech House, Boston, Mass, USA, 3rd edition, 2005. 


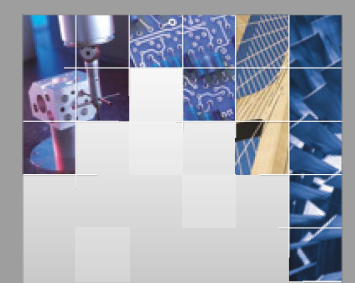

\section{Enfincering}
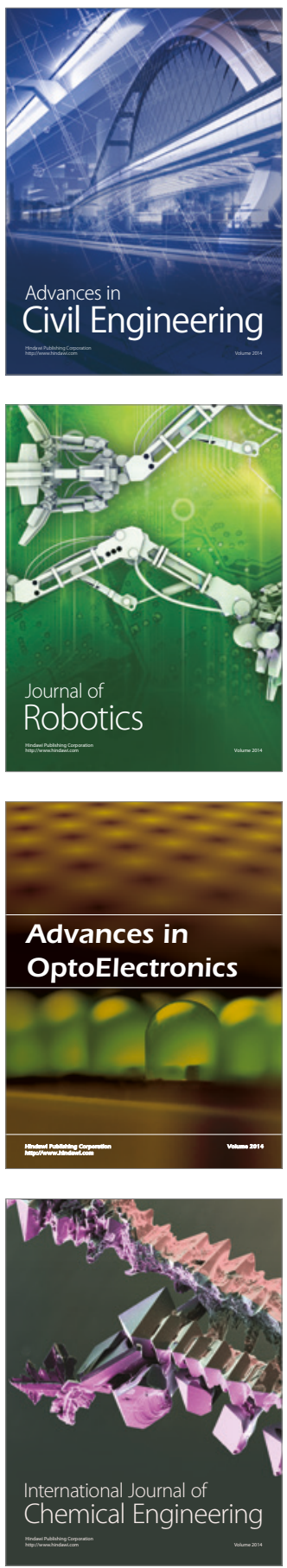

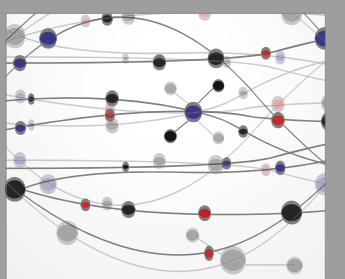

The Scientific World Journal

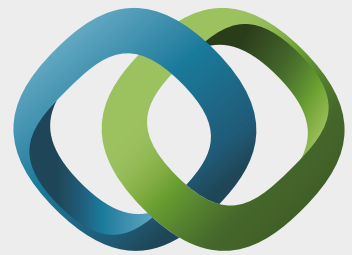

\section{Hindawi}

Submit your manuscripts at

https://www.hindawi.com
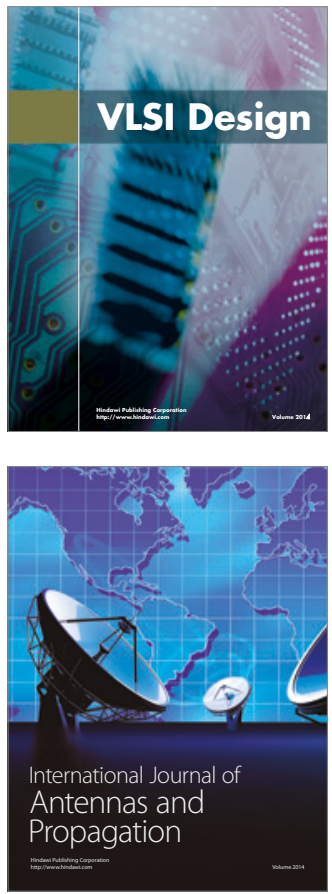

\section{Rotating}

Machinery
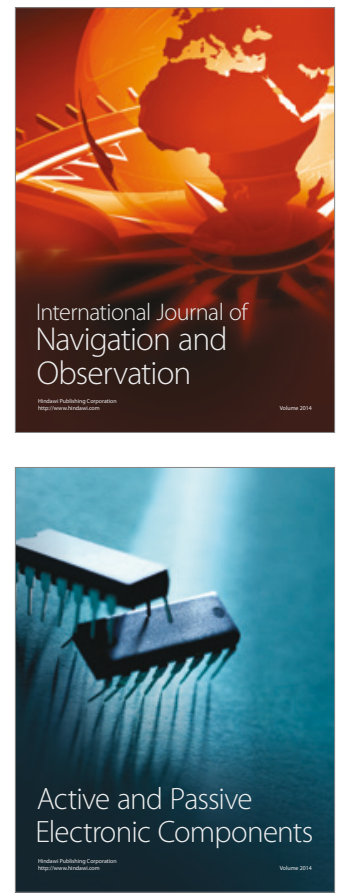
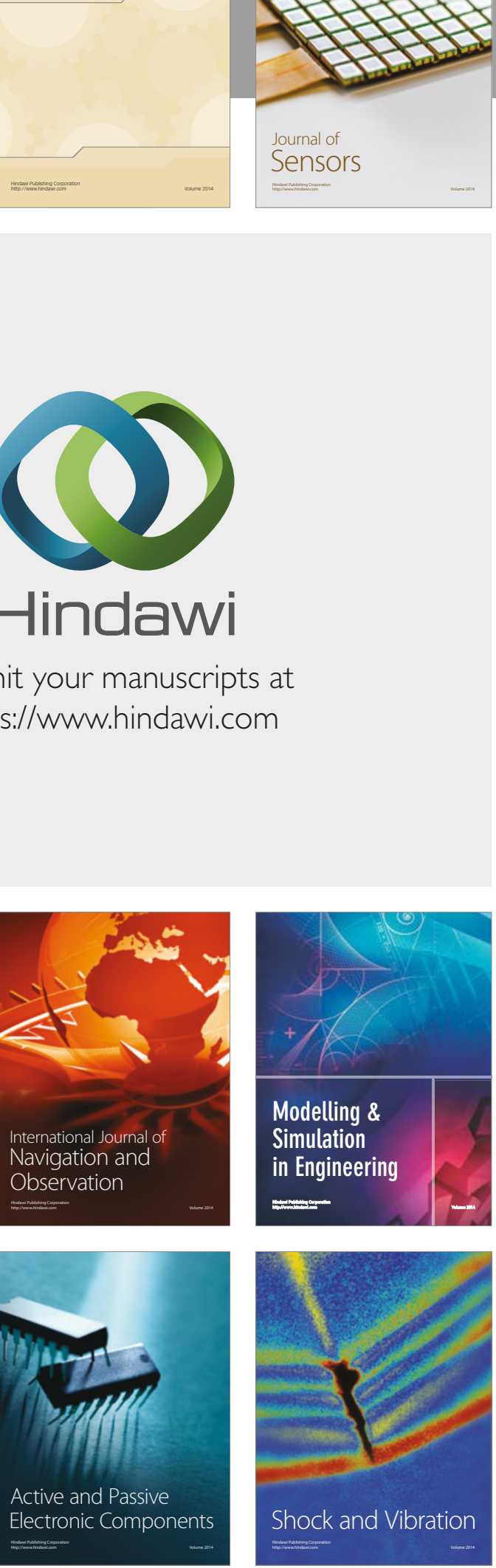
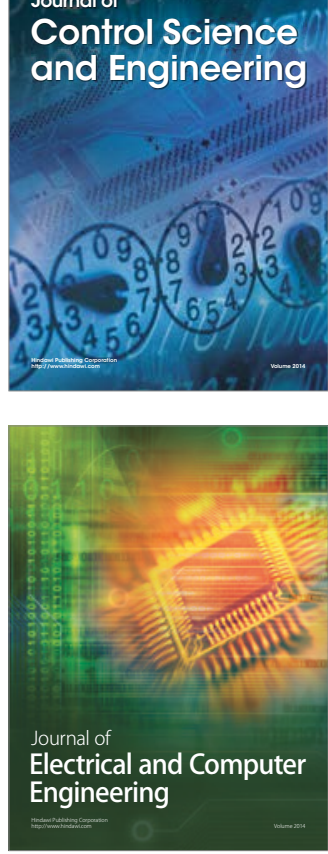

Distributed

Journal of

Control Science

and Engineering
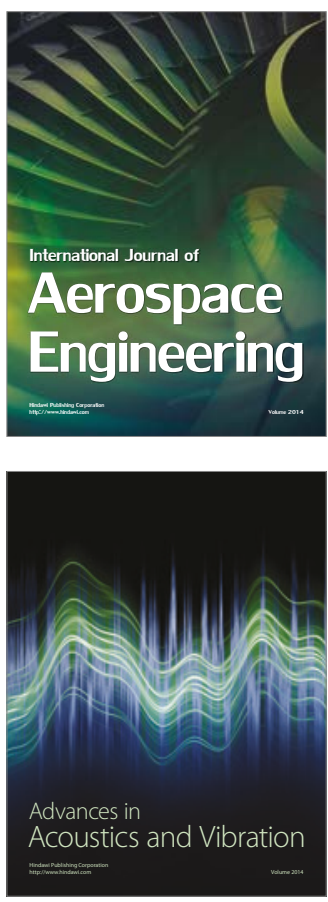

Sensor Networks 